Switching Costs and the Gittins Index

Author(s): Jeffrey S. Banks and Rangarajan K. Sundaram

Source: Econometrica, Vol. 62, No. 3 (May, 1994), pp. 687-694

Published by: The Econometric Society

Stable URL: http://www.jstor.org/stable/2951664

Accessed: 18-03-2016 18:07 UTC

Your use of the JSTOR archive indicates your acceptance of the Terms \& Conditions of Use, available at http://www.jstor.org/page/ info/about/policies/terms.jsp

JSTOR is a not-for-profit service that helps scholars, researchers, and students discover, use, and build upon a wide range of content in a trusted digital archive. We use information technology and tools to increase productivity and facilitate new forms of scholarship. For more information about JSTOR, please contact support@jstor.org. 


\title{
SWITCHING COSTS AND THE GITTINS INDEX
}

\author{
By Jeffrey S. Banks and Rangarajan K. Sundaram ${ }^{1}$
}

\section{INTRODUCTION}

THE THEOREM OF GiTTINS AND JONES (1974) is, perhaps, the single most powerful result in the literature on Bandit problems. This result establishes that in independent-armed Bandit problems with geometric discounting over an infinite horizon, all optimal strategies may be obtained by solving a family of simple optimal stopping problems that associate with each arm an index known as the dynamic allocation index or, more popularly, as the Gittins index. Importantly, the Gittins index of an arm depends solely on the characteristics of that arm and the rate of discounting, and is otherwise completely independent of the problem under consideration. These features simplify significantly the task of characterizing optimal strategies in this class of problems. ${ }^{2}$

The purpose of this paper is to examine the extent to which the Gittins-Jones theorem remains valid when the cost of switching between arms is possibly nonzero, i.e., to determine whether suitably defined index strategies continue to remain optimal in this case. The need to include switching costs arises primarily from economic considerations. Such an extension is, perhaps, of especial interest in a labor market setting where the Bandit framework has found wide applicability, ${ }^{3}$ but appears more generally important. Indeed, it is difficult to imagine a relevant economic decision problem in which the decision-maker may costlessly move between alternatives. ${ }^{4}$

As our framework of analysis, we use a generalized version of Whittle (1982) with arm-specific costs of switching. It is obvious that in the most inclusive case where there is a cost $c_{i j}$ for switching from an arm $i$ to another arm $j$, there cannot exist an optimal index strategy with the index on an arm depending solely on the arm's characteristics. We consider therefore, the more restrictive case where the cost of switching away from an arm (resp. to an arm) are independent of the arm to which (resp. from which) the switch is made. In principle, at least, this leaves open the possibility that an optimal index strategy may exist.

Unfortunately, our main result is negative. We show that, in general, it is not possible to define indices which have the property that the resulting index strategy is optimal on the domain of all Bandit problems with switching costs. Indeed, this remains true even if attention is restricted to that subset of the domain in which the cost of switching is a given (nonzero) constant. From one point of view, this nonexistence may not appear very

\footnotetext{
${ }^{1}$ We are very grateful to Andy McLennan for several helpful conversations. We would also like to thank Martin Hellwig and two referees for their comments. The first author gratefully acknowledges financial support provided by the Sloan Foundation and the NSF. An earlier version of this paper was written during the second author's sabbatical at the California Institute of Technology, and he would like to thank them for their hospitality.

${ }^{2}$ See, e.g., Whittle (1982), or Banks and Sundaram (1992).

${ }^{3}$ See, e.g., Mortensen (1985). See also Banks and Sundaram (1992), who provide a list of other applications in economics and political science.

${ }^{4}$ It is somewhat surprising to note, therefore, that the literature on switching costs consists only of a few stray papers. Examples include Kolonko and Benzing (1983) who study the special case of a two-armed Bandit with one known arm, where the other arm generates rewards according to a Bernoulli distribution with unknown parameters; and Agrawal, et al. (1988) who study the existence of asymptotically efficient adaptive allocation rules (in the sense of Lai and Robbins (1985)) in the presence of switching costs. See also the recent contributions of Feldman and Spagat (1993) on the impact of switching costs in a general model of optimal Bayesian learning.
} 
surprising, for the Gittins index is known to be nonrobust in some (other) directions. For instance, the stationarity of the underlying problem is very important: Berry and Fristedt (1985) show that geometric discounting is a necessary condition for the validity of the Gittins-Jones theorem. On the other hand, the addition of switching costs does not affect the model's stationarity, and certainly, there is no a priori reason to expect lack of robustness in this direction. In particular, Weitzman (1979) shows that optimal index strategies do exist in the closely related "Pandora's Box" problem, where there is a nonzero cost to be paid the first time an alarm is used, but subsequent visits to the arm are free, even if one has switched away to another arm in the interim.

Our proof of the nonexistence of an optimal index uses a reductio ad absurdum approach: we assume that an optimal index strategy does exist, derive some of the properties it must satisfy, and show that these properties are not mutually consistent. The intuition underlying our construction is quite straightforward. Consider an $n$-armed Bandit problem, and suppose the decision maker is currently on some arm (the incumbent arm). If, in the optimal continuation, there is any possibility of switching back to the incumbent arm after leaving it (depending on, say, the realizations from the other arms), then the index on the incumbent arm must depend nontrivially on the cost of switching (back) to it, since a higher cost of coming back should make the decision-maker more reluctant to leave the arm. If, on the other hand, coming back to this arm is a zero-probability event (say, because the worst realizations on the other arms would still dominate the present incumbent) the arm's index must be independent of the cost of switching back to it. Thus, the index on an incumbent arm changes depending on whether we compare it to an arm whose payoff prospects are known for certain, or to an arm whose payoffs involve some uncertainty, and operationalizing this idea furnishes the required contradiction.

The remainder of this paper is organized as follows. Section 2 provides a description of Bandit problems, introduces switching costs, and defines the notion of an optimal index in this case. Section 3 formalizes the intuition of the previous paragraph in proving the nonexistence of an optimal index under switching costs, both in general and in the restricted case where attention is limited to problems having an a priori given and fixed switching cost.

\section{BANDIT PROBLEMS}

\subsection{The Standard Framework}

Our description of the standard Bandit framework in this paper is, of necessity, terse. We also keep the technical exposition at a relatively informal level. For omitted details, we refer the reader to Whittle (1982).

An independent-armed Bandit problem with geometric discounting (hereafter, simply Bandit problem) is defined by the following objects: ${ }^{5}$

1. A set $N=\{1, \ldots, n\}$ of arms of the Bandit, where $n$ is a positive integer.

2. A tuple $F_{i}=\left(X_{i}, r_{i}, Q_{i}\right)$ for each arm $i$ where:

(a) $X_{i}$, a subset of some Polish (i.e., complete, separable, metric) space, describes the set of possible states of arm $i$, with generic element $x_{i}$;

(b) $r_{i}: X_{i} \rightarrow \mathfrak{R}$ is a bounded measurable function describing the instantaneous reward from arm $i$; and

${ }^{5}$ Our description follows Whittle (1982). In the "classical" version of the Bandit problem, as used for instance, by Berry and Fristedt (1985), the states of arm $i$ would correspond to the set of possible beliefs the decision-maker may have regarding the "true" distribution of rewards from arm $i$; the transition probabilities are implicitly defined by the map taking prior beliefs and observed rewards into posterior beliefs. 
(c) $Q_{l}$ represents a family of transition probabilities on $X_{i}$, i.e., for each $x_{i} \in X_{i}$, $Q_{l}\left(\cdot \mid x_{i}\right)$ is a probability distribution on $X_{i}$, and for each fixed Borel subset $D$ of $X_{i}, Q_{i}(D \mid \cdot)$ is a measurable mapping from $X_{i}$ into $[0,1]$.

3. A discount factor $\rho \in[0,1)$.

The Bandit problem has the following interpretation. In each period $t=0,1,2, \ldots$, of an infinite horizon, a decision-maker must decide which arm of the Bandit is to be employed in that period, given the vector of states $\left(x_{1}^{t}, \ldots, x_{n}^{t}\right)$ at the beginning of that period. This decision is made with full knowledge of the history of the problem to date. If arm $i$ is chosen in period $t$, two things happen. First, the decision-maker receives a reward of $r_{l}\left(x_{l}^{t}\right)$. Second, the state of arm $i$ transits to its period $(t+1)$-value $x_{i}^{t+1}$ according to the (conditional) probability distribution $Q_{i}\left(\cdot \mid x_{i}^{t}\right)$. The states of all other arms remain frozen, so that we have $x_{j}^{t+1}=x_{j}^{t}$ for all $j \neq i$. The decision-maker discounts future rewards by the factor $\rho \in[0,1)$, and aims to maximize total discounted expected reward over the infinite horizon.

More formally, for any $t \geqslant 0$, a $t$-history $h_{t}$ for the problem is a description of the state of each arm in each period up to $t$, the action taken in each of those periods, and the period- $t$ state. Let $H_{0}=X_{1} \times \cdots \times X_{n}$, and let $H_{t}$ be the set of all possible histories up to $t$. A strategy $\sigma$ for the decision-maker is a rule that recommends the arm to be played at any point in time as a function of the history up to that point, i.e., it is a sequence of maps $\left\{\sigma_{t}\right\}$, where for each $t \geqslant 0, \sigma_{t}$ is a measurable map from $H_{t}$ into $N$.

Each strategy $\sigma$ defines in the obvious way an expected $t$ th period reward, denoted $r_{t}[x]$, from each initial state $x=\left(x_{1}, \ldots, x_{n}\right)$ and for each $t$. The total worth of $\sigma$ from $x$, denoted $W(\sigma)(x)$, is then defined as $W(\sigma)(x)=\sum_{t=0}^{\infty} \rho^{t} r_{t}[x]$. A strategy $\sigma^{*}$ is an optimal strategy if its worth is maximal amongst all strategies, i.e., if $W\left(\sigma^{*}\right)(x)=\sup _{\sigma} W(\sigma)(x)$ for all $x$.

Standard arguments from dynamic programming (see, for instance, Whittle (1982)) establish that optimal strategies exist in this problem, and, indeed, that stationary Markovian optimal strategies ${ }^{6}$ exist. The breakthrough achieved by Gittins and Jones (1974) lies in showing that a particularly simple class of strategies-those defined through the Gittins index - actually suffice to obtain all optimal strategies. We turn now to a brief description of this result. In the sequel, $\rho$ is assumed fixed at some level in $[0,1)$, and all dependence on $\rho$ is suppressed.

\subsection{The Gittins Index}

The Gittins index on an arm $i$, whose characteristics are given by $F_{i}=\left(X_{i}, r_{i}, Q_{i}\right)$, is obtained by the following procedure. Let $m \in \mathfrak{R}$ be given. Consider the stopping problem in which in each period (given that the terminal reward $m$ has not yet been accepted) the decision-maker must choose between playing arm $i$ for one more period, and stopping and accepting the terminal reward $m$. Routine arguments show that the value $V\left(x_{i}, F_{i} ; m\right)$ of this problem is well-defined and finite from any initial state $x_{i} \in X_{i}$. The Gittins index on arm $i$, denoted by $\mu\left(x_{i}, F_{i}\right)$ is then defined by

$$
\mu\left(x_{i}, F_{i}\right)=\inf \left\{m \mid V\left(x_{i}, F_{i} ; m\right)=m\right\} .
$$

Since $r_{i}$ is bounded by assumption, it follows that for large $m$, we have $V\left(\cdot ; F_{i}, m\right)=m$, while for $-m$ large, $V\left(x_{i}, F_{i} ; m\right)$ is independent of $m$. Thus, the Gittins index is well-defined. The importance of this index lies in the following result. Let $\left\{N,\left(F_{i}\right)_{i \in N}\right\}$ be an arbitrary Bandit problem.

${ }^{6} \mathrm{~A}$ stationary Markovian strategy $\sigma$ is a strategy under which the period $t$ action depends solely on the period $t$ state vector $x^{t}=\left(x_{1}^{t}, \ldots, x_{n}^{t}\right)$, but not on how or when the state was reached. Such a strategy can evidently be represented by a measurable function $g: X_{1} \times \cdots \times X_{n} \rightarrow N$, with the interpretation that $g(x)$ is the action recommended by the strategy when the state is $x$. 
TheOREM 1 (Gittins and Jones (1974)): The optimal selections at the state $\left(x_{1}, \ldots, x_{n}\right)$ in the Bandit $\left\{N,\left(F_{i}\right)_{i \in N}\right\}$ are those arms $i$ for which $\mu\left(x_{i}, F_{i}\right)=\max \left\{\mu\left(x_{j}, F_{j}\right) \mid j \in N\right\}$.

Equivalently, the Gittins-Joines theorem may be stated as follows: a strategy $\sigma$ for a Bandit problem $\left\{N,\left(F_{i}\right)_{i \in N}\right\}$ is an optimal strategy if, and only if, the set of histories on which the recommendations of $\sigma$ differ from the Gittins index-maximal arms after that history has probability zero.

\subsection{Switching Costs in the Bandit Framework}

The most general way to introduce switching costs in the Bandit framework is to assume that there exists a cost $c_{i j}$ for switching from arm $i$ to $\operatorname{arm} j, i, j \in\{1, \ldots, N\}$. It is clear, however, that no index can then be defined which is such that the resulting strategy is optimal, if the index for an arm is to depend on that arm alone. The formulation we use here, therefore, is more specialized, and one that leaves open the possibility, at least at the intuitive level, that optimal index strategies may exist.

Specifically, we associate with each arm a pair $\left(c_{i}, d_{i}\right)$ of real numbers where (i) $c_{i}$ is the cost of switching to arm $i$ (from any arm), and (ii) $d_{i}$ is the cost of switching away from arm $i$ (to any arm). Thus, if a switch occurs from $\operatorname{arm} i$ to arm $j$, the total cost paid is $d_{i}+c_{j}$.

To avoid further complicating notation, in the sequel the tuple $F_{i}$ describing arm $i$ is to be understood as including the vector $\left(c_{i}, d_{i}\right)$ also.

When switching costs are admitted, the state of the Bandit problem in any period cannot, except at the very beginning, be adequately described by just the vector $\left(x_{1}, \ldots, x_{n}\right)$. Rather, it is also important to know the arm that was in use in the period immediately preceding. (We will henceforth refer to this arm as the arm "currently in use.") Defining $\Delta=X_{1} \times \cdots \times X_{n} \times N$, and letting $x$ denote the vector $\left(x_{1}, \ldots, x_{n}\right)$, routine arguments now show that the value function $V: \Delta \rightarrow \mathfrak{R}$ for this problem satisfies the Bellman optimality equation at each $(x, j) \in \Delta$ :

$$
V(x, j)=\max _{i \in N} L_{i} V(x, j)
$$

where, for $i \neq j$,

$$
L_{i} V(x, j)=r_{i}\left(x_{i}\right)-c_{i}-d_{j}+\rho \int V\left(x_{-i}, \hat{x}_{i}, i\right) Q_{i}\left(d \hat{x}_{i} \mid x_{i}\right)
$$

while

$$
L_{j} V(x, j)=r_{j}\left(x_{j}\right)+\rho \int V\left(x_{-j}, \hat{x}_{j}, j\right) Q_{j}\left(d \hat{x}_{j} \mid x_{j}\right)
$$

and that any measurable selection from the correspondence of maximizers of (2.2) constitutes a stationary Markovian optimal strategy. In order, however, to examine the existence of optimal index strategies, we must first define the notion of an index for this problem. We turn to this now.

\subsection{The Index with Switching Costs}

As with the Gittins index, we shall define an index on a generic arm $i$ to be any function obtainable solely from the characteristics $F_{i}$ of arm $i$. However, if we require 
the index to depend on $F_{i}$ alone, then simple examples show that index strategies cannot be optimal. Consider the following:

EXAMPLE 1: Let $N=\{1,2\} ; X_{1}=X_{2}=[0,1] ; r_{1}=r_{2}=r$, where $r(x)=x$, for all $x \in$ $[0,1] ; Q_{1}=Q_{2}=Q$, where $Q(1 \mid x)=1-Q(0 \mid x)=x$, for all $x \in[0,1] ; d_{1}=d_{2}=d>0$, while $c_{1}=c_{2}=0$; and, finally, let $\rho$ be any value in $(0,1)$.

Consider any indices $\lambda\left(x_{i}, F_{t}\right)$ for the arms. Since the arms are identical up to the initial state, we must have $\lambda\left(\cdot, F_{1}\right)=\lambda\left(\cdot, F_{2}\right)=\lambda(\cdot, F)$, say, where $F_{1}=F_{2}=F$. It is evident that the attractiveness of an arm is increasing in the value of the initial state, so that, if at all $\lambda$ is to be optimal, it must be increasing on [0,1]. In particular, we must have $\lambda(x, F)>\lambda(0, F)$ for any $x>0$.

But $\lambda(x, F)>\lambda(0, F)$ for all $x>0$ is inconsistent with the optimality of $\lambda$. For, suppose we had $0=x_{1}<x_{2}$, and $d>x_{2} /(1-\delta)$. Suppose further that the decision-maker is currently on arm 1. It is clear then that the unique optimal policy is simply to stay with arm 1 forever, but $\lambda$ recommends a shift to arm 2 , which is strictly suboptimal.

The reason this example "works" is that in requiring the index to depend on $F_{l}$ alone, we have omitted the crucial bit of information about whether arm $i$ was the arm that was in use in the previous period. For, it is obvious that in comparing two otherwise identical arms, one of which was used in the previous period, the one which was in use must necessarily be more attractive than the one which was idle. This motivates the following definition.

Definition: An index in the presence of switching costs is any function $\lambda$ which specifies for a generic arm $i$, a value $\lambda\left(x_{l}, F_{l}, s_{i}\right)$, where $F_{l}$ denotes the characteristics of arm $i, x_{l}$ is the current state of arm $i$, and $s_{i} \in\{0,1\}$ is a variable that specifies whether $\left(s_{i}=1\right)$, or not $\left(s_{t}=0\right), i$ is the arm currently in use.

An index $\lambda$ induces in each Bandit problem $\left\{N,\left(F_{t}\right)_{t \in N}\right\}$, a strategy $\sigma(\lambda)$ in the obvious manner: let $x$ be the vector of initial states. In period $0, \sigma(\lambda)$ plays any of the arms $i$ for which $\lambda\left(x_{i}, F_{i}, 0\right)=\max \left\{\lambda\left(x_{j}, F_{j}, 0\right) \mid j \in N\right\}$. For each subsequent period $t$, let $x^{t}$ denote the vector of states at the beginning of period $t$, and $i(t-1)$ the arm that was used in period $(t-1)$. Then, in period $t, \sigma(\lambda)$ plays any of the arms $i$ for which $\lambda\left(x_{i}, F_{l}, s_{l}\right)=\max \left\{\lambda\left(x_{j}, F_{j}, s_{j}\right) \mid j \in N\right\}$, where $s_{l}=1$ iff $i=i(t-1)$.

Finally, an index $\lambda$ is said to be an optimal index in the presence of switching costs if $\sigma(\lambda)$ is optimal in every Bandit problem $\left\{N,\left(F_{i}\right)_{\imath \in N}\right\}$.

\section{THE NON-EXISTENCE OF AN OPTIMAL INDEX}

We show in this section that an optimal index does not exist in the presence of switching costs. Our argument consists of two parts. First, we will show that any Bandit problem with costs of switching "from" (and, possibly, also costs of switching "to"), is equivalent to another problem in which there are only costs of switching "to." We will then consider the case where the only switching costs are costs of switching "to," and show that in a series of steps, if an optimal index does exist a contradiction must result, completing the proof.

So let a Bandit $B=\left\{N,\left(F_{i}\right)_{l \in N}\right\}$ be given. Define another Bandit $B^{*}=\left\{N,\left(F_{i}^{*}\right)_{i \in N}\right\}$ from $B$ as follows: for each $i$, let $X_{t}^{*}=X_{i} ; r_{i}^{*}\left(x_{i}\right)=r_{i}\left(x_{t}\right)+(1-\rho) d_{i} ; Q_{i}^{*}=Q_{i} ; c_{t}^{*}=$ $c_{i}+d_{i}$; and, finally, $d_{i}^{*}=0$. We will show that the Bandits $B$ and $B^{*}$ are equivalent.

Indeed, this is almost immediate. Viewed as dynamic programming problems, $B$ and $B^{*}$ have the same state and action spaces, hence the same strategy spaces. Moreover, since the transition probabilities also coincide, a given strategy induces the same distribution on infinite histories in either problem. Thus, it suffices to show that a given history yields the same reward in either problem, or more specifically, that the net 
reward from a given arm over the periods of its contiguous use is the same in either problem. To see that this is true, observe that, in essence, the only difference between Bandits $B$ and $B^{*}$ is that in Bandit $B$, a cost of $d_{i}$ is paid every time a switch away from arm $i$ occurs, whereas in Bandit $B^{*}, d_{i}$ is paid "in advance" when the switch to arm $i$ occurs, but an additional reward of $(1-\rho) d_{i}$ is received every period arm $i$ is in use. If, therefore, arm $i$ is used for $t$ contiguous periods before a switch to another arm occurs, the present value of the total switching cost paid in the Bandit $B$ will be $c_{i}+\rho^{t} d_{i}$. In Bandit $B^{*}$, the total cost will be $c_{i}+d_{i}$; but an additional reward of $(1-\rho) d_{i}$ is received in each of $t$ periods, so that the net cost is $c_{i}+d_{i}\left(1-\sum_{s=0}^{t-1} \rho^{s}(1-\rho)\right)=c_{i}+d_{i} \rho^{t}$, which is exactly the same as in the Bandit $B$. Thus, the sums of discounted rewards in the two problems differ only by the cost of switching away from the initial arm, and the equivalence of $B$ and $B^{*}$ follows.

We now proceed to the second part of our proof, which consists essentially of formalizing the intuitive arguments given in the Introduction. We suppose from now on that there are only costs of switching "to," and that an optimal index, denoted $\lambda$, does exist in this case. Since all the Bandits we shall consider from this point on involve arms of only two types, we simplify notation as follows. First, for $x \in[0,1]$ and $c \geqslant 0$, let $\left[x \delta_{1}+(1-x) \delta_{0}, c\right]$ denote an arm with state space $[0,1]$ and initial state $x$; reward function $r(x)=x$; transition probabilities $Q(1 \mid x)=1-Q(0 \mid x)=x$ for all $x \in[0,1]$; and switching cost $c$. Second, let $\left[\delta_{a}, c\right]$ denote an arm with switching cost $c$, that pays a reward of $a \in \Re$ in each period with certainty.

In this notation, $\lambda\left(\left[x \delta_{1}+(1-x) \delta_{0}, c\right] ; s\right)$ and $\lambda\left(\left[\delta_{a}, c\right] ; s\right)$ will denote, respectively, the value of the optimal index on the arms $\left[x \delta_{1}+(1-x) \delta_{0}, c\right]$ and $\left[\delta_{a}, c\right]$, at the state $s \in\{0,1\}$. Recall that $s=1$ denotes that the arm is currently in use.

Claim 1: Any index $\lambda$ that is optimal in the presence of switching costs must be a strict monotone transformation of an index $\hat{\lambda}$ which satisfies

$$
\begin{aligned}
& \hat{\lambda}\left(\left[\delta_{a}, c\right] ; 1\right)=a, \\
& \hat{\lambda}\left(\left[\delta_{a}, c\right] ; 0\right)=a-c(1-\rho),
\end{aligned}
$$

for any values of $a$ and $c$.

Proof: Consider the situation where there are only two arms. Suppose arm $i(=1,2)$ pays $a_{i}$ for certain, and that the cost of switching to arm $i$ is $c_{i}$. Finally, suppose that the decision-maker is currently on the first arm.

It is trivial to see that the uniquely optimal strategy is to stay with arm 1 forever if $a_{1}>a_{2}-c_{2}(1-\rho)$; that picking either arm initially and staying with it forever is optimal if $a_{1}=a_{2}-c_{2}(1-\rho)$; and that switching to arm 2 and staying there forever is uniquely optimal if $a_{1}<a_{2}-c_{2}(1-\rho)$. Since $\hat{\lambda}$ satisfies (3.1)-(3.2), it is evidently optimal in this class of problems. Since any optimal index $\lambda$ must also be optimal on this limited class of problems, it is immediate that any such index must be a strict monotone transformation of $\hat{\lambda}$, establishing the claim.

Q.E.D.

By Claim 1, we may henceforth assume, without loss, that the optimal index $\lambda$ that is presumed to exist satisfies (3.1)-(3.2). The following claim now establishes the impossibility of an optimal index.

Claim 2: There is no consistent way to define an index $\lambda$ on arms of the form $\left[x \delta_{1}+(1-x) \delta_{0}, c\right]$ if the resulting strategy is to be invariably optimal. Consequently, an optimal index cannot exist. 
Proof: Consider the class of Bandit problems in which there are two arms, the first of the sort $\left[\delta_{a}, c_{1}\right]$, and the second of the sort $\left[x \delta_{1}+(1-x) \delta_{0}\right]$. Suppose that the decisionmaker is on the first arm.

Let $\nu$ be defined by

$$
\nu\left(x, c_{1}, c_{2}\right)=\frac{x-(1-\rho)\left[(1-x) \rho c_{1}+c_{2}\right]}{1-\rho(1-x)} .
$$

Some simple, direct calculation shows that if $(1-\rho)\left(c_{1}+c_{2}\right) \leqslant x$ and $a=\nu\left(x, c_{1}, c_{2}\right)$, this Bandit problem has exactly two optimal continuations: playing arm 1 forever, or switching initially to arm 2, and switching back to arm 1 if, and only if, the state of arm 2 moves to zero. Therefore, either arm is an optimal initial selection under, these circumstances, and we must have

$$
\lambda\left(\left[x \delta_{1}+(1-x) \delta_{0}, c_{2}\right] ; 0\right)=\lambda\left(\left[\delta_{\nu\left(x, c_{1}, c_{2}\right)}, c_{1}\right] ; 1\right)=\nu\left(x, c_{1}, c_{2}\right),
$$

when $(1-\rho)\left(c_{1}+c_{2}\right) \leqslant x$.

It is easy to see that (3.4) is already in contradiction to the existence of an optimal index if the costs of switching are allowed to differ across arms, since it requires $\lambda\left(\left[x \delta_{1}+(1-x) \delta_{0}, c_{2}\right] ; 0\right)$ to depend nontrivially on $c_{1}{ }^{7}$ Thus, it only remains to be shown that an optimal index cannot exist even when the cost of switching to any arm is fixed at some constant (nonzero) level $c .{ }^{8}$ We restrict attention to this case in the sequel. Rewriting (3.4) for this case by setting $c_{1}=c_{2}=c$, we see that if $2 c(1-\rho) \leqslant x$, then our hypothetical optimal index $\lambda$ must satisfy

$$
\lambda\left(\left[x \delta_{1}+(1-x) \delta_{0}, c\right] ; 0\right)=\nu(x, c, c)=\frac{x-c(1-\rho)[1+\rho(1-x)]}{1-\rho(1-x)} .
$$

Now consider the same two-armed Bandit (with the first arm of type $\left[\delta_{a}, c\right]$ and the second of type $\left.\left[x \delta_{1}+(1-x) \delta_{0}, c\right]\right)$, but suppose that the decision-maker is initially on the second arm. Direct calculation shows that when

$$
a=\mu(x, c):=\frac{x}{(1-\rho(1-x))}+c(1-\rho),
$$

either arm is an optimal initial selection. Thus, we must have $\lambda\left(\left[x \delta_{1}+(1-x) \delta_{0}, c\right] ; 1\right)=$ $\lambda\left(\left[\delta_{\mu(x, c), c}\right] ; 0\right)$, and so, by Claim 1 and the presumed optimality of $\lambda$,

$$
\lambda\left(\left[x \delta_{1}+(1-x) \delta_{0}, c\right] ; 1\right)=\frac{x}{1-\rho(1-x)} .
$$

We will show that (3.5) and (3.7) are not consistent with the optimality of $\lambda$. To this end, consider a two-armed Bandit, where the first arm is specified by $\left[x \delta_{1}+(1-x) \delta_{0}, c\right]$, and the second arm by $\left[y \delta_{1}+(1-y) \delta_{0}, c\right]$. Assume that the decision-maker is currently on the first arm. As is readily checked, the uniquely optimal strategy is to pick the first arm (and then to switch to the second if the state of the first moves to 0 ) when the

${ }^{7}$ A precise version of this contradiction may be derived as follows. Fix any $x \in(0,1)$ and $\rho \in(0,1)$, and suppose $c_{2}=x /[2(1-\rho)]$. Note that $(3.4)$ applies whenever $c_{1} \leqslant x /[2(1-\rho)]$. When $c_{1}=x /$ [2(1- $\rho)], \lambda\left(\left[x \delta_{1}+(1-x) \delta_{0}, c_{2}\right] ; 0\right)=\nu\left(x, c_{1}, c_{2}\right)=x / 2$ by (3.4) and Claim 1. On the other hand, when $c_{1}=0, \lambda\left(\left[x \delta_{1}+(1-x) \delta_{0}, c_{2}\right] ; 0\right)=\nu\left(x, c_{1}, c_{2}\right)=x /[2(1-\rho+\rho x)]$, which is evidently different from $x / 2$.

${ }^{8}$ The possibility of existence of an optimal index in this case was raised by a referee. 
following conditions are met:

$$
\begin{aligned}
& y>c(1-\rho)>x, \\
& \frac{x}{1-\rho(1-x)}>y-c(1-\rho) .
\end{aligned}
$$

Thus, we must have

$$
\lambda\left(\left[x \delta_{1}+(1-x) \delta_{0}, c\right] ; 1\right)>\lambda\left(\left[y \delta_{1}+(1-y) \delta_{0}, c\right] ; 0\right)
$$

whenever (3.8)-(3.9) hold.

But it is easy to construct examples where these inequalities are inconsistent with the definition of $\lambda$ from (3.5) and (3.7). For instance, let $\rho=c=1 / 2, x=3 / 17$, and $y=27 / 50$. Then, $y>1 / 4=c(1-\rho)>x$, so (3.8) is met. Moreover, the left-hand side of (3.9) reduces to $2 x /(1+x)=3 / 10$, while the right-hand side of (3.9) is $29 / 100$, so (3.9) is also met. Therefore, (3.10) must hold.

However, from (3.7) we have

$$
\lambda\left(\left[x \delta_{1}+(1-x) \delta_{0}, c\right] ; 1\right)=\frac{3 / 17}{1-(1 / 2)(14 / 17)}=\frac{3}{10},
$$

while (since $y>1 / 2=2 c(1-\rho)),(3.5)$ implies

$$
\lambda\left(\left[y \delta_{1}+(1-y) \delta_{0}, c\right] ; 0\right)=\frac{(27 / 50)-(123 / 400)}{1-(1 / 2)(23 / 50)}=\frac{93}{308} .
$$

Since $93 / 308>3 / 10,(3.11)$ and (3.12) imply $\lambda\left(\left[y \delta_{1}+(1-y) \delta_{0}, c\right] ; 0\right)>\lambda\left(\left[x \delta_{1}+\right.\right.$ $\left.\left.(1-x) \delta_{0}, c\right] ; 1\right)$, a contradiction to (3.10), completing the proof. Q.E.D.

Dept. of Economics, Harkness Hall, University of Rochester, Rochester, NY 14627-0156, U.S.A.

Manuscript received March, 1992; final revision received August, 1993.

\section{REFERENCES}

Agrawal, R., M. V. Hegde, and D. Teneketzis (1988): "Asymptotically Efficient Adaptive Allocation Rules for the Multiarmed Bandit Problem with Switching Costs," IEEE Transactions on Optimal Control, 33, 899-906.

Banks, J. S., And R. K. Sundaram (1992): Denumerable-Armed Bandits," Econometrica, 60, 1071-1096.

Berry, D. A., ANd B. Fristedt (1985): Bandit Problems: Sequential Allocation of Experiments. London: Chapman and Hall.

Feldman, M., and M. Spagat (1993): “Optimal Learning with Costly Adjustment,” Working Paper No. 93-10, Department of Economics, Brown University.

GitTins, J. C., AND D. M. Jones (1974): "A Dynamic Allocation Index for the Sequential Allocation of Experiments," in Progress in Statistics, ed. by J. Gani et al. Amsterdam: North-Holland, pp. 241-266.

Kolonko, M., AND H. Benzing (1983): "The Sequential Design of Bernoulli Experiments Including Switching Costs," unpublished manuscript; cited in Berry and Fristedt (1985).

LaI, T. L., AND H. RobBins (1985): "Asymptotically Efficient Adaptive Allocation Rules," Advances in Applied Mathematics, 6, 4-22.

MortenSEN, D. (1985): "Job Search and Labor Market Analysis," in Handbook of Labor Economics, Vol. II, ed. by O. Ashenfelter and J. Layard. New York: North Holland, pp. 849-919.

Weitzman, M. (1979): "Optimal Search for the Best Alternative," Econometrica, 47, 641-654.

Whitrle, P. (1982): Optimization Over Time: Dynamic Programming and Stochastic Control, Vol I. New York: Wiley. 